support provided by his Fellowship of St. John's College, Cambridge.

\section{References}

1. J. T. Dehn, Phys. Letters 29A (1969) 132.

2. R. V. Pound and G. A. Rebka Jr., Phys. Rev. Letters
$4(1960) 274$.

3. B. D. Josephson, Phys. Rev. Letters 4 (1960) 341.

4. C. Moller, The Theory of Relativity (Clarendon Press, Oxford, 1952) p. 62.

5. H. F auenfelder, The Morssbauer effect (W. A. Benjamin, Inc., New York, 1963) pp. 20-21.

6. Reference 4, pp, 48-51, 247 and 258-263.

7. C. W. Sherwin, Phys. Rev. 120 (1960) 17.

\title{
EFFECT OF RECOIL ON THE VELOCITY DISTRIBUTION OF METASTABLE ATOMS PRODUCED BY ELECTRON IMPACT*
}

\author{
J. C. PEARL, D. P. DONNELLY and J. C. ZORN
}

Randall Laboratory of Physics, University of Michigan, Ann Arbor, Michigan 48104, USA

Received 3 September 1969

\begin{abstract}
We have shown both experimentally and by a simple kinematic argument that the velocity distribution in a beam of metastable atoms produced by electron bombardment of ground state atoms deviates strongly from the velocity distribution of the ground state atoms.
\end{abstract}

The velocity distribution of metastable atoms often enters explicitly into the analysis of resonance [1] and collision $[2,3]$ experiments done with atomic beams. However, there have been few direct measurements and no correct theoretical description of the velocity distribution in a beam of metastable

* Research supported in part by the National Aeronautics and Space Administration.

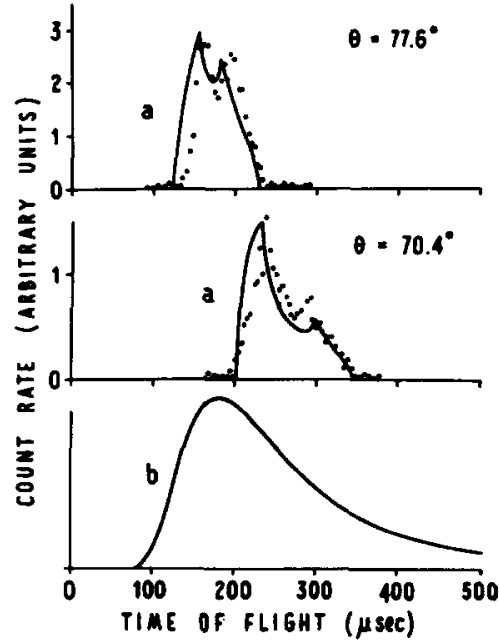

Fig. 1. Time-of-flight spectra for helium. Points represent experimental data. Curves a are theoretical accounting for the angular divergence of the incoming, ground state beam, assuming $E-E *=0.75$ eV. Curve b is theoretical if recoil effects are neglected. 
atoms formed in the common experimental arrangement where ground state atoms in a collimated beam are excited by a transverse beam of electrons.

In our experiment a pulsed electron beam is directed at right angles $\left(\Phi=90^{\circ}\right)$ through a collimated beam of thermal energy helium atoms. The metastable atoms are detected [4] with an electron multiplier (Bendix Channeltron) which can be rotated about the interaction region in the plane defined by the incoming electron and atom beams. Samples of data taken at two different angles $\theta$ (angles measured from direction of electron beam) are plotted as points in fig. 1. Striking features of these results, when compared with what would be found if recoil effects were negligible (curve b in fig. 1) include the narrow. ness of the distributions, the presence of two peaks, and the sensitivity to deflection angle of the position of the distribution.

The principal features of these distributions can be understood from kinematic considerations. If the gas beam effuses ideally from a thermal source (most probable speed $\alpha$ ), if the ground state beam is perfectly collimated, if the detector is small, and if the electron scattering is s-wave, then conservation of momentum and energy determine the following velocity distribution for the metastable atoms in the plane of the incident beams

$$
f(v)=\frac{v^{2}}{V} \frac{v_{\mathrm{O}_{+}}^{2} \exp \left(-v_{\mathrm{O}_{+}}^{2} / \alpha^{2}\right)+v_{0-}^{2} \exp \left(-v_{\mathrm{O}_{-}}^{2} / \alpha^{2}\right)}{\left[V^{2}-\left\{(m / M) u_{0} \sin \Phi-v \sin (\Phi-\theta)\right\}^{2}\right]^{\frac{1}{2}}}
$$

for $v_{\min }<v<v_{\max }$, where

$$
v_{\min }=\left[\frac{m}{M} u_{0} \sin \Phi \pm V\right] \operatorname{cosec}(\Phi-\theta) \quad\left\{\begin{array}{l}
\theta<\Phi \\
V<\left(m u_{0} / M\right) \sin \Phi
\end{array}\right.
$$

and

$$
v_{0 \pm}=v \cos (\Phi-\theta)-\frac{m}{M} u_{\mathrm{o}} \cos \Phi \pm\left\{V^{2}-\left[\frac{m}{M} u_{\mathrm{o}} \sin \Phi-v \sin (\Phi-\theta)\right]^{2}\right\}^{\frac{1}{2}}
$$

Here $V=\left[2 m\left(E-E^{*}\right)\right]^{\frac{1}{2}} / M ; m$ and $M$ are the masses of the electron and atom respectively; $E^{*}$ is the energy of the metastable state; and $u_{\mathrm{o}}$ and $E$ are the speed and kinetic energy, respectively, of the incident electron. The distribution $f(v)$, which is zero outside the limits given in eq. (2), is in disagreement with functions previously presented $[7,8]$. In the present experiment the detector subtends only a very small angle $\left(\Delta \theta \approx 0^{\circ} 8^{\prime}\right)$, but the divergence of the incoming beam $\left(\Delta \Phi \approx 3^{\circ}\right)$ is significant. A numerical integration of eq. (2) over the beam divergence yields curves a of fig. 1.

The present study of velocity distributions in beams of metastable atoms should facilitate the interpretation of experiments such as the fine and hyperfine structure measurements in $2^{2} S_{\frac{1}{2}}$ hydrogen $[1,5]$, measurements of electron impact excitation of $n=2$ states in helium [6], time-of-flight studies of repulsive molecular states [7], and analysis of the kinetic properties of single and multi-component gases [8].

We are indebted to $R$ A. Heppner, E. S. Fry and W. L. Williams for their assistance and encouragement. The importance of velocity distributions to the measurement of atomic fine structure was emphasized to us by R. T. Robiscoe

\section{References}

1. W. E. Lamb Jr. and R. C. Retherford, Phys. Rev, 79 (1950) 549.

2. R. F. Stebbings, W. L. Fite, D. G. Hummer and R. T. Brackmann, Phys. Rev. 119 (1960) 1939

3. B. Bederson, Atomic interactions, Part A, eds. B. Bederson and W. L. Fite (Academic Press, Inc., New York, 1968) pp. 89-95.

4. D. P. Donnelly, J. C. Pearl, R. A. Heppner and J. C. Zorn, Rev. Sci. Inst. (to be pubished). See also pp. 85-87 of ref. 3.

5. J.W. Heberle, H. A. Reich and P. Kusch, Phys. Rev, 101 (1956) 612;

R. T. Robiscoe, Phys. Rev. 138 (1965) A22 and Phys. Rev. 168 (1968) 4. 
6. H. K. Holt and R. V. Krotkov, Phys. Rev. 144 (1966) 82;

L. Vriens, T. F. M. Bonsen and J. A. Smit, Physica 40 (1968) 229.

7. M. Leventhal, R. T. Robiscoe and K. R. Lea, Phys. Rev. 158 (1967) 49;

R. Freund and W. Klemperer, J. Chem. Phys. 47 (1967) 2897;

R. Clampitt and A. S. Newton, J. Chem. Phys. 50 (1969) 1997.

8. J. B. French and J.W. Locke in Rarefied gas dynamics, ed. C. L. Brundin (Academic Press, Inc., New York, 1967) p. 1461;

D. A. Crosby and J. C. Zorn, J. Vac. Sci. Tech. 6 (1969) 82.

\title{
A KINETIC EQUATION FOR DILUTE POLYATOMIC GASES
}

\author{
A. TIP
}

FOM-Instituut voor Atoom-en Molecuulfysica, Amsterdam, The Netherlands

Received 22 August 1969

\begin{abstract}
A kinetic equation is obtained for dilute polyatomic gases which is applicable to rapidly varying processes. It constitutes a generalization of an equation derived previously by Waldmann and Snider, the latter being valid when the level spacing between internal states are sufficiently large.
\end{abstract}

From the quantum mechanical BBGKY hierarchy equations for the one and two particle density operators $\rho_{1}^{(1)}(t)$ and $\rho_{12}^{(2)}(t)$

$$
\begin{aligned}
& \partial_{t} \rho_{1}^{(1)}(t)=-\mathrm{i} L_{1}^{(1)} \rho_{1}^{(1)}(t)-\mathrm{i} \underset{2}{\operatorname{tr}} L_{12}^{(\mathrm{int})} \rho_{12}^{(2)}(t) \\
& \partial_{t} \rho_{12}^{(2)}(t)=-\mathrm{i} L_{12}^{(2)} \rho_{12}^{(2)}(t)-\mathrm{i} \operatorname{tr}_{3} L_{12 / 3}^{(\mathrm{int})} \rho_{123}^{(3)}(t)
\end{aligned}
$$

one derives the following approximate equation of motion for the pair correlation function $g_{12}^{(2)}(t)=$ $=\rho_{12}^{(2)}(t)-\rho_{1}^{(1)}(t) \rho_{2}^{(1)}(t)$

$$
\partial_{t} g_{12}^{(2)}(t)=-\mathrm{i} L_{12}^{(2)} g_{12}^{(2)}(t)-\mathrm{i} L_{12}^{(\mathrm{int})} \rho_{1}^{(1)}(t) \rho_{2}^{(1)}(t)
$$

Here contributions from the trace parts in (1) and (2) are neglected. The Liouville operators have the following meaning: $L_{1}^{(1)}$ is derived from the free one particle Hamiltonian, $L_{12}^{(0)}$ from the free two particle one. $L_{12}^{(2)}=L_{12}^{(0)}+L_{12}^{(\text {int) }}$ contains in addition the two particle interaction potential whereas $\left.L_{12}^{(\text {int }}\right)_{3}$ is related to the interaction of the pair $(1,2)$ with a third particle. Substituting the formal solution

$g_{12}^{(2)}(t)=\exp \left[-\mathrm{i} L_{12}^{(2)}\left(t-t_{\mathrm{o}}\right)\right] g_{12}^{(2)}\left(t_{\mathrm{o}}\right)-\mathrm{i} \int_{0}^{t-t_{\mathrm{o}}} \mathrm{d} s \exp \left[-\mathrm{i} L_{12}^{(2)} s\right] L_{12}^{(\mathrm{int})} \rho^{(1)}(t-s) \rho^{(1)}(t-s)$,

of eq. (3) into eq. (1) obtains :

$$
\begin{aligned}
\partial_{t} \rho_{1}^{(1)}(t) & =-\mathrm{i} L_{1}^{(1)} \rho_{1}^{(1)}(t)-\mathrm{i} \operatorname{tr} L_{12}^{(\mathrm{int})} \exp \left[-\mathrm{i} L_{12}^{(2)}\left(t-t_{\mathrm{o}}\right)\right] g_{12}^{(2)}\left(t_{\mathrm{o}}\right)+ \\
& -\int_{0}^{t-t_{\mathrm{o}}} \mathrm{d} s \underset{2}{\operatorname{tr}} L_{12}^{(\mathrm{int})} \exp \left[-\mathrm{i} L_{12}^{(2)} s\right] L_{12}^{(\mathrm{int})} \rho_{1}^{(1)}(t-s) \rho_{2}^{(1)}(t-s)-\mathrm{i} \underset{2}{\operatorname{tr}} L_{12}^{(\mathrm{int})} \rho_{1}^{(1)}(t) \rho_{2}^{(1)}(t)
\end{aligned}
$$

